\title{
Cooling the chest while keeping the abdomen warm: the best of both worlds in multi-organ preservation from controlled donation after circulatory death donors?
}

\author{
Dirk Van Raemdonck a,b, ${ }^{*}$, Laurens J. Ceulemans a,b, Ina Jochmans c,d and Arne Neyrinck e,f
}

a Department of Thoracic Surgery, University Hospitals Leuven, Leuven, Belgium

b Department of Chronic Diseases and Metabolism, Katholieke Universiteit Leuven, Leuven, Belgium

c Department of Abdominal Transplant Surgery, University Hospitals Leuven, Leuven, Belgium

d Department of Microbiology, Immunology, and Transplantation, Katholieke Universiteit Leuven, Leuven, Belgium

e Department of Anesthesiology, University Hospitals Leuven, Leuven, Belgium

$f$ Department of Cardiovascular Sciences, Katholieke Universiteit Leuven, Leuven, Belgium

* Corresponding author. Department of Thoracic Surgery, University Hospital Gasthuisberg, Herestraat 49, 3000 Leuven, Belgium. Tel: +32-16-34-68-23;

fax: +32-16-34-68-24; e-mail: dirk.vanraemdonck@uzleuven.be (D. Van Raemdonck).

Preservation of donor organs by cold fluid perfusion followed by rapid recovery (RR) and static cold storage prior to transplantation has been standard practice for $>5$ decades since its conception by our pioneers [1]. At lower temperatures, catabolic enzymes are suppressed and cell metabolism is slowed down, protecting the organ from post-transplant dysfunction once reperfused. This strategy has evolved to the standard of care for organ preservation from both donors after neurological determination of death (DNDDs) and donors after circulatory determination of death (DCDDs).

As a result of the rising demand for transplant organs, the percentage of DCDD, especially those recovered under controlled circumstances after withdrawal from life-sustaining therapy (WLST), has risen in the last decade in countries where this practice is permitted [2]. Controlled donors after circulatory determination of death (CDCDD) organs, however, are subjected to a variable degree of hypotension and hypoxia during the agonal phase after WLST, followed by a mandatory period of warm ischaemia after asystole until cold flush and a subsequent period of cold ischaemia during storage and transportation. The outcomes of abdominal CDCDD organ transplantation are variable. CDCDD kidney recipients are subjected to an increased risk of delayed graft function [3] while liver transplantation is associated with a higher incidence of primary non-function and ischaemic type biliary lesions [4]. In contrast, CDCDD donor lungs appear to tolerate the WLST process well with no post-transplant outcome differences compared to DNDD lung recipients [5]. Warming is the antonym of cooling. Dynamic organ preservation by machine perfusion was demonstrated to be successful at different temperatures. Normothermic regional perfusion (NRP), in which extracorporeal membrane oxygenation (ECMO) technology is used to restore blood flow inside the deceased donor itself to minimize warm ischaemic organ damage, has emerged as a new procurement technique following the pioneering work in uncontrolled DCDDs in Spain [6]. Soon after, this technique was explored in other countries for in situ preservation of abdominal CDCDD organs (A-NRP) [7] or in combination with thoracic organs (TA-NRP) first described by the Papworth team [8].

In this issue of the journal, authors from the University Hospital Puerta de Hierro Majadahonda in Madrid report the findings of a single-centre retrospective analysis comparing outcome between 2 comparable recipient cohorts ( $n=14$ each) transplanted between June 2013 and December 2019 with lungs from CDCDDs whose abdominal organs were recovered by A-RR versus A-NRP techniques [9]. After certification of the donor's death and rapid sternotomy, all donor lungs were preserved in the 
same standard manner with cold anterograde perfusion, followed by RR and static cold storage. Functional warm ischaemic time [2] of lungs was comparable between both cohorts (21 vs 25 min, respectively). No significant differences in early outcome were identified with comparable rates of primary graft dysfunction, intensive care unit and hospital stays, in-hospital mortality and 1-year survival. The authors concluded that this 'dual temperature' technique is feasible and safe for both thoracic and abdominal organs. Moreover, outcome after lung transplantation was not influenced by the recovery technique for CDCDD abdominal organs.

Two issues are important to discuss. First, the outcome in abdominal organ recipients was not reported in detail. It is unclear whether these organs may have suffered by the early retrieval of lungs while the deceased body is still connected to ECMO. The authors stated that no abdominal organs were lost after lung extraction. A-NRP is technically more complex. Once lungs are removed, significant blood loss may occur from the dissected mediastinum or pleural surface. Meticulous hemostasis is important.

In addition, lungs may suffer transdiaphragmatic rewarming during A-NRP. Alternatively, lungs could remain topically cooled inside the chest or continuously ventilated in situ [10] while the abdominal organs are being preserved and assessed with A-NRP. Second, in young donors qualifying for CDCDD cardiac transplantation, combining the technique of RR for the heart and lungs with A-NRP for the preservation of liver and kidneys does not seem to fit. TA-NRP would then become the best alternative technique allowing conversion of a non-heart-beating procedure into a heart-beating procedure. It is, however, important to understand that with TA-NRP, lungs will not receive anterograde blood perfusion through the pulmonary arteries until the arrested heart starts beating again. Meanwhile, lungs will receive limited flow of oxygenated blood via the bronchial circulation originating from the descending aorta. Therefore, in the setting of A-NRP using a clamp on the descending aorta, or in cases where the heart does not generate sufficient cardiac output during TA-NRP, lungs will not be perfused while remaining in the chest at body temperature, although tolerating warm ischaemia well up to 120 min. Administration of heparin directly into the right atrium and pulmonary trunk on opening the pericardium is advised to prevent widespread thrombosis in the lungs if not given previously upon WLST [8]. The Madrid group is to be congratulated with their results and important contribution. The authors demonstrated that ECMO for in situ warm perfusion of abdominal organs does not negatively impact donor lung quality. This is an important message that may help in convincing other lung transplant teams not to decline a potential CDCDD lung offer once the abdominal team has decided to use A-NRP for the assessment and preservation of liver and kidneys. A-NRP is not a contraindication for simultaneous CDCDD lung retrieval by cold flush perfusion.

Organ retrieval practice in CDCDDs is likely to change considerably as an increasing number of centres are now introducing A-NRP to preserve abdominal organs. The best preservation strategy for lungs is still not clear and the role of TA-NRP needs further investigation. This procedure has several advantages in terms of allowing functional assessment and resuscitation of all organs after death, including the heart. TA-NRP turns CDCDD multi-organ retrieval back into a 'heart-beating' procedure. In this way, the standard technique of cold fluid perfusion for the preservation of all CDCDD organs becomes possible, turning this into a more controlled procedure. The paper by Tanaka et al. adds to the evidence that recovery techniques at different temperatures can be safely combined in cDCDDs: cooling the chest while keeping the abdomen warm. Further studies are needed to compare outcome in CDCDD lung transplant recipients between RR and TA-NRP.

\section{Funding}

D.V.R. is supported by the Broere Charitable Foundation. 


\section{REFERENCES}

[1] Belzer FO, Southard JH. Principles of solid-organ preservation by cold storage. Transplantation 1988;45:673-6.

[2] Thuong M, Ruiz A, Evrard P, Kuiper M, Boffa C, Akhtar MZ et al. New classification of donation after circulatory death donors definitions and terminology. Transpl Int 2016;29:749-59.

[3] Summers DM, Johnson RJ, Allen J, Fuggle SV, Collett D, Watson CJ et al. Analysis of factors that affect outcome after transplantation of kidneys donated after circulatory death in the UK: a cohort study. Lancet 2010; 376:1303-11.

[4] Jay CL, Lyuksemburg V, Ladner DP, Wang E, Caicedo JC, Holl JL et al. Ischemic cholangiopathy after controlled donation after cardiac death liver transplantation: a meta-analysis. Ann Surg 2011;253:259-64.

[5] Van Raemdonck D, Keshavjee S, Levvey B, Cherikh WS, Snell G, Erasmus M et al. Donation after circulatory death in lung transplantation-five year follow-up from ISHLT Registry. J Heart Lung Transplant 2019;38: 1235-45.

[6] Fondevila C, Hessheimer AJ, Ruiz A, Calatayud D, Ferrer J, Charco R et al. Liver transplant using donors after unexpected cardiac death: novel preservation protocol and acceptance criteria. Am J Transplant 2007; 7: 1849-55.

[7] Oniscu GC, Randle RV, Muiesan P, Butler AJ, Currie IS, Perera MT et al.In situ normothermic regional perfusion for controlled donation after circulatory death-The United Kingdom experience. Am J Transplant 2014;14:2846-54.

[8] Tsui SSL, Oniscu GC. Extending normothermic regional perfusion to the thorax in donors after circulatory death. Curr Opin Organ Transplant 2017;22:245-50.

[9] Tanaka S, Campo-Canaveral de la Cruz JL, Crowley Carrasco S, Romero Roman A, Hoyos Meja L, Naranjo Gomez JM et al. Effect on the donor lungs of using abdominal normothermic regional perfusion in controlled donation after circulatory death. Eur J Cardiothorac Surg 2020; in press.

[10] Palleschi A, Tosi D, Rosso L, Zanella A, De Carlis R, Zanierato M et al. Successful preservation and transplant of warm ischaemic lungs from controlled donors after circulatory death by prolonged in situ ventilation during normothermic regional perfusion of abdominal organs. Interact CardioVasc Thorac Surg 2019;29:699-705. 